members of the $F c \gamma$ receptor $(F c \gamma R)$ family. Fc $\gamma$ RIIA is the most abundantly expressed Fc $\gamma \mathrm{R}$ in human blood circulation. However, mice do not express Fc $\gamma$ RIIA, and murine platelets are completely devoid of Fc $\gamma$ Rs.

Methods To model IC-mediated response more accurately, as they occur in humans, transgenic Fc $\gamma$ RIIA ( $\mathrm{Fc} \gamma \mathrm{RIIA}^{\mathrm{TGN}}$ ) mice expressing FcyRIIA on platelets and certain leukocytes, were used in this study. As acute model, we intravenously injected ICs in FcyRIIA ${ }^{\text {null }}$ and Fc $\gamma$ RIIA $^{\text {TGN }}$ mice and monitored mouse reaction and platelet activation. To model platelet response to chronic exposure to ICs, we backcrossed NZB mice with Fc $_{\text {RIIA }}{ }^{\text {TGN }}$ mice, generating NZB::Fc $\gamma$ RIIA ${ }^{\text {TGN }}$ mice and crossed the mice with NZW mice, thus generating NZB:: NZW::Fc $\gamma R^{\text {RIIA }}{ }^{\text {TGN }}$ and NZB::NZW::Fc $\gamma$ RIIA $^{\text {null }}$ mice. Platelet activation was monitored through time in these mice.

Results Platelet activation by acute exposure to ICs through a mechanism requiring expression of platelet Fc $\gamma$ RIIA resulted in the induction of systemic shock. IC-driven shock was dependent on release of serotonin from platelet dense granules secondary to platelet outside-in signaling by $\alpha \operatorname{IIb} \beta 3$ and its ligand fibrinogen. On FcyRIIA activation, platelets underwent sequestration, but surprisingly they returned in the blood circulation with emptied granules after activation. Strikingly, reminiscent observations were made in lupus NZB::NZW::Fc $\gamma$ RIIA $^{\text {TGN }}$ mice. We found significant platelet activation and circulating degranulated platelets, uniquely in mice expressing Fc $\gamma$ RIIA.

Conclusions Platelet activation in IC-mediated pathogenesis is well recognized. In lupus patients, platelets are found activated in blood circulation, however, to what extend ICs and Fc $\gamma$ RIIA contribute to platelet activation was unknown. Here, we showed that the expression of FcyRIIA is critical to adequately examine platelet role in lupus.

Acknowledgements This work was supported by a Foundation grant from the Canadian Institutes of Health Research (CIHR) to EB. PRF is recipient of a tier 1 Canada Research Chair on Systemic Autoimmune Rheumatic Diseases. NT and IM are recipient of fellowships from The Arthritis Society.

\section{Al-06 LUPUS NEPHRITIS IS LINKED TO DYSBIOSIS, INCREASED GUT LEAKINESS AND IMMUNITY TO AN INTESTINAL COMMENSAL LACHNOSPIRACAEA SPECIES}

${ }^{1}$ Gregg J Silverman*, 'Doua F Azzouz, ${ }^{2}$ Brad H Rovin, ${ }^{3}$ Roberto Caricchio, ' ${ }^{1}$ ill P Buyon, ${ }^{4}$ Alexander Alekseyenko. ${ }^{1}$ Department of Medicine, New York University School of Medicine, New York, NY, USA; ${ }^{2}$ Division of Nephrology, Ohio State University Medical Center, Columbus, OH, USA; ${ }^{3}$ Medicine/Rheumatology, Temple University, Philadelphia, PA, USA; ${ }^{4}$ Medical University of South Carolina, Charleston, SC, USA

\subsection{6/lupus-2018-Ism.6}

Background A transmissible agent has long been suspected in the pathogenesis of SLE, yet the potential contribution of the human intestinal microbiome has been little examined. We therefore characterized the gut microbiota of patients with SLE, with special interest in those with lupus nephritis (LN).

Methods Blood and fecal samples from SLE patients were obtained, with strict inclusion/exclusion of criteria. Fecal $16 \mathrm{~S}$ rDNA sequencing, as well as cytokine and autoantibody assays were performed. In addition, sera from two independent lupus cohorts were studied for validation. Biomarkers of gut leakiness were assessed.

Results Compared to controls, the intestinal microbiome from SLE patients $(n=61)$ showed decreased species richness diversity with reductions in taxonomic complexity most pronounced in those with high disease activity. Notably, SLE patients had an overall 5-fold greater representation of a species in the Lachnospiracaea family of obligate anaerobic Grampositive cocci, with reciprocal contractions of two other commensal species with putative protective properties. Abundance of the Lachnospiracaea species correlated with serum IgG to a cell wall component, postulated to represent a lipoglycan, from a strain of this same species $(p=0.002, n=61$, Spearman) but not with 7 other strains. There was also a significant direct correlation between SLEDAI scores and levels of these circulating anti-strain IgG antibodies $(p=0.02, n=48)$. Levels of antibodies to strain-specific bacterial antigen, treated with RNAse/DNAse/proteinase K, were significantly higher in those with active nephritis at time of sampling compared to SLE without renal activity (Cohort $1 \mathrm{p}=0.01 \mathrm{n}=48$; Cohort 2 $\mathrm{p}=0.006, \mathrm{n}=28$, Mann-Whitney). Levels of serum IgG antistrain antibodies also significantly correlated with high-titer serum IgG to native DNA $(\mathrm{p}<0.0001, \mathrm{n}=27)$, and inversely correlated with C3 and C4 levels. High titers of these antibacterial antibodies were associated with active Class III, IV and V (overlap) LN (Cohort 3).

Conclusions These findings suggest a novel paradigm for the pathogenesis of $\mathrm{LN}$ in which a common intestinal commensal bacteria may contribute to the immune-complex mediated disease process, with features akin to poststreptococcal GN but without outward signs and symptoms of clinical infection.

Acknowledgements Judith and Stuart Colton Foundation, NIAMS- P50 AR070591.

\section{Al-07 A NEW B CELL EFFECTOR PATHWAY WITH DEFECTIVE NEGATIVE REGULATION OF TLR7 SIGNALING IN HUMAN SLE}

${ }^{1}$ Scott A Jenks, ${ }^{1}$ Kevin S Cashman, ${ }^{2}$ Urko M Marigorta, 'S Sam Lim, ${ }^{3}$ Michelle Petri, ${ }^{4}$ Jennifer Anolik, 'Ignacio Sanz*. 'Department of Medicine, Division of Rheumatology, Lowance Center for Human Immunology, Emory University, Atlanta, Georgia, USA; ${ }^{2}$ School of Biology and Center for Integrative Genomics, Georgia Institute of Technology, Atlanta, GA, USA; ${ }^{3}$ Hopkins Lupus Center, Department of Medicine, Johns Hopkins University, Baltimore, MD, USA; ${ }^{4}$ Department of Medicine, Division of Allergy, Immunology and Rheumatology, University of Rochester Medical Center, Rochester, New York, USA

\subsection{6/lupus-2018-Ism.7}

Background B cell homeostasis is perturbed in SLE patients. in particular, many patients have a large expansion of IgDCD27- B cells (DN). The DN population is heterogeneous for CXCR5 expression, and CXCR5- DN2 are the majority population in SLE patients but not in HCD (figure 1A). To further understand how these expanded cells differ from other B cells subsets and how they may be dysregulated in SLE, we phenotypically and functionally characterized DN2 in SLE patients and healthy control donors (HCD).

Methods B cells subsets were quantified by flow cytometry in HCD and two separate cohorts of lupus patients. Purified DN2 and other B cell subsets were flow sorted and transcriptionally analyzed using RNA sequencing. Toll-like-receptor 7 (TLR7) signaling after stimulation with R848 was measured by staining with anti-phospho-tyrosine specific anti-ERK. Antibody secreting cell differentiation was induced using in vitro stimulation of sorted B cell subsets with a combination of TLR7 and cytokines.

Results DN2 were only a minor B cell subset in HCD (less than $5 \%$ ) but were elevated in $20 \%$ of cohort 1 patients and $60 \%$ of cohort 2 (figure $1 \mathrm{~B}$ ). In the patients with the largest 
expansions almost all B cells (80\%) had a DN2 phenotype. DN2 cells predominate in African-American patients with active disease and nephritis, anti-Smith and anti-RNA autoantibodies. Among B cells, they express the highest levels of a Tbet/Zeb2 transcriptional network characteristic of effector $T$ cells and lack the negative TLR regulator, TRAF5. Consistent with this transcriptional profile DN2 are hyper-responsive to TLR7. Moreover, DN2 cells share with activated naïve cells phenotypic, functional features, and a very similar transcriptome. When stimulated in vitro DN2 readily differentiated into plasma cells and produced autoantibodies.

Conclusions DN2 and activated naïve represent a separate B cell lineage with a distinct origin and function as they differed from other B cells subsets both in uniquely expressing several genes and a lack expression of other genes. This study defines a distinct differentiation fate of human autoreactive naïve $B$ cells into effector plasma cell precursors with innate hyperresponsiveness to stimuli relevant to SLE, establishes the components and prominence of extrafollicular B cell activation in this disease, and identifies new therapeutic targets.
A

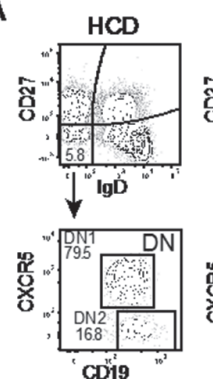

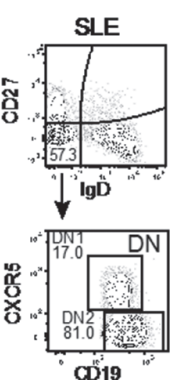

B

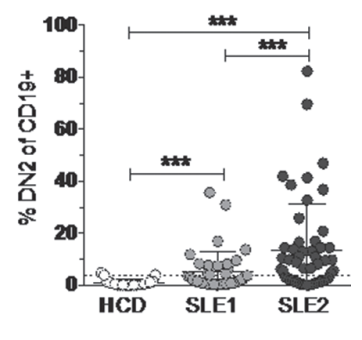

Abstract Al-07 Figure 1 A) representative flow profiles showing that IgD-/CD27-/CXCR5-/CD19++ DN2 B cells are rare in HCD but expanded in many SLE patients. B) the frequency of DN2 for HCD and 2 SLE patient cohorts as percentage of CD19+

\section{AI-08 CHARACTERIZATION OF HUMAN AGE-ASSOCIATED B CELLS (ABCS) IN NORMAL AND LUPUS PERIPHERAL BLOOD}

Jennifer L Barnas*, Jennifer Albrecht, Nida Meednu, Lin Gao, Mary O'Connell, R John Looney, Jennifer H Anolik. University of Rochester, Rochester, NY, USA

\subsection{6/lupus-2018-Ism.8}

Background Age-associated B cells (ABCs) are associated with autoantibody production in lupus-like mice. This population is expanded upon exposure to self-antigen and interferon- $\gamma$. $\mathrm{ABCs}$ in mice are identified as CD11c+, CD21- and T-bet+. However, the characterization of these cells and factors promoting their generation in human SLE is unclear. The purpose of this study was to define ABCs in human SLE patients and their association with interferon (IFN) status.

Methods Peripheral blood leukocytes were purified by density gradient centrifugation from 26 lupus patients with a range of disease activity $(n=10$ with flaring disease, $n=16$ controlled disease) and 6 normal healthy donors. Surface and T-bet intracellular antibody staining was analyzed in a blinded fashion by flow cytometry. A putative population of ABCs was defined as CD3 - CD19+ CD11c+ CD21- cells. Samples were divided into groups regardless of disease status based upon percentage of $\mathrm{B}$ cells with putative $\mathrm{ABC}$ phenotype. B cell subset analysis was completed on the groups expressing minimal $(\leq 2 \%)$, intermediate $(2 \%-7 \%)$, or high $(\geq 8 \%)$ levels of ABCs. In addition, the $\mathrm{B}$ cell subset distribution (switched memory, unswitched memory, total naïve, and $\mathrm{CD} 27-\mathrm{IgD}$ - double negative) and phenotypic markers (CXCR3, T-bet, CD24, IgD, and CD27) of putative $\mathrm{ABCs}$ were assessed. IFN- $\alpha$, IFN- $\beta$, and IFN- $\lambda$ levels were quantitated by ELISA from sera drawn at the same time.

Results Five samples were identified as having high putative ABC levels (range $8.4 \%-20.5 \%$ of B cells, mean 14.9\%). These CD11c+CD21- CD19+CD3 - cells were predominantly CD24-CD27- IgD-. Across all 32 samples, the percentage of putative $\mathrm{ABCs}$ positively correlated with percentage of T-bet $+\mathrm{B}$ cells $\left(\mathrm{r}=0.819, \mathrm{p}=5.4 \times 10^{-9}\right)$, IgD- CD27(double negative) B cells $(\mathrm{r}=0.59, \mathrm{p}=0.0003)$, and CD24double negative cells $\left(r=0.74, p=8.72 \times 10^{-7}\right)$ with a high degree of statistical significance. Paired serum samples had a range of type I and type IIII interferon levels (mean +SEM for all samples): $6.2 \pm 2.1 \mathrm{pg} / \mathrm{m}$ (IFN- $\alpha$ ), $4.0 \pm 0.6 \mathrm{pg} / \mathrm{ml}$ (IFN$\beta$ ), and $44.1 \pm 9.6 \mathrm{pg} / \mathrm{ml}$ (IFN- $\lambda 1)$. Analysis including correlation between $\mathrm{B}$ cell subsets, interferon status, clinical features and disease activity is in progress.

Conclusions An expanded putative ABC population (CD11c+ CD21- CD19+ CD3-) was identified in a subset of human peripheral blood samples. This subset is positively correlated with IgD-CD27-CD24- B cells (DN2 cells), whose expansion has been described previously in lupus patients.

\section{\begin{tabular}{|l|l}
\hline Al-09 T FOLLICULAR HELPER (TFH) CELLS ARE INCREASED IN \\
\hline
\end{tabular} ASYMPTOMATIC ANTI-NUCLEAR ANTIBODY (ANA) ${ }^{+}$ INDIVIDUALS AND APPEAR TO PLAY A ROLE IN EPITOPE SPREADING}

1,2,3,4 Joan Wither* ${ }^{1}$ Nan-Hua Chang, ${ }^{3,4,5,6}$ Sindhu R Johnson, ${ }^{3}$ Waleed Hafiz, ${ }^{1,2}$ Kieran Manion, ${ }^{1,2}$ Dario Ferri, ${ }^{1}$ Ariana Karanxha, ${ }^{1}$ Babak Noamani, 'Dennisse Bonilla, ${ }^{1}$ Sina Rusta-Sellehy, ${ }^{7}$ Larissa Lisnevskaia, ${ }^{3,4}$ Zahi Touma, ${ }^{8,9}$ Earl Silverman, ${ }^{3,4}$ Arthur Bookman, ${ }^{1}$ Carolina Landolt-Marticorena, ${ }^{1,2}$ Yuriy Baglaenko. ${ }^{1}$ Krembil Research Institute, University Health Network, Toronto, Canada; ${ }^{2}$ Department of Immunology, University of Toronto, Toronto, Canada; ${ }^{3}$ Division of Rheumatology, University Health Network, Toronto, Canada; ${ }^{4}$ Department of Medicine, University of Toronto, Toronto, Canada; ${ }^{5}$ Division of Rheumatology, Mount Sinai Hospital, Toronto, Canada; ${ }^{6}$ Institute of Health Policy, Management and Evaluation, University of Toronto, Toronto, Canada; ${ }^{7}$ Lakeridge Heath Services, Oshawa, Canada; ${ }^{8}$ Division of Rheumatology, Sick Children's Hospital, Toronto, Canada; ${ }^{9}$ Department of Pediatrics, University of Toronto, Toronto, Canada

\subsection{6/lupus-2018-Ism.9}

Background The diagnosis of Systemic Autoimmune Rheumatic Diseases (SARD), including Systemic Lupus Erythematosus (SLE), relies on the presence of ANAs, many of which can be detected years before clinical manifestations. However, ANAs are also seen in healthy individuals most of whom will not develop SARD. A number of cellular immune changes are seen in SARD, and thus could constitute potential biomarkers/ treatment targets for SARD, however it is not known at what point in disease progression these develop.

Methods Healthy $\mathrm{ANA}^{-}$controls $(n=32)$ and $\mathrm{ANA}^{+}(\geq 1: 160$ by immunofluorescence) participants with no (asymptomatic $\mathrm{ANA}^{+}, \mathrm{n}=61$ ), at least one (UCTD, $\mathrm{n}=35$ ), or meeting SARD classification criteria $(n=59)$ were recruited. Peripheral blood cellular immunological changes were assessed by flow cytometry. 